\title{
Avaliação de um programa computadorizado para intervenção fônica na dislexia do desenvolvimento
}

\author{
Darlene Godoy de Oliveira - Universidade Presbiteriana Mackenzie, São Paulo, Brasil \\ Katerina Lukasova - Universidade Cruzeiro do Sul, São Paulo, Brasil \\ Elizen Coutinho de Macedo ${ }^{1}$ Universidade Presbiteriana Mackenzie, São Paulo, Brasil
}

\begin{abstract}
Resumo
A teoria do Déficit Fonológico explica as trocas e déficits de consciência fonológica em sujeitos com dislexia do desenvolvimento. Intervenções focadas nesse processamento contribuem para melhora da leitura. Este estudo verificou a eficácia do Software Alfabetização Fônica Computadoriz̧ada na promoção de consciência fonológica e correspondências grafofonêmicas em disléxicos. Vinte crianças disléxicas foram divididas em dois grupos (GI e GII) e foram pareadas por idade, sexo e escolaridade. Os participantes realizaram provas de consciência fonológica e habilidades de leitura e de escrita. Os participantes do GI frequentaram 16 sessões de intervenção e, após o período, os grupos foram reavaliados. Houve aumento significativo no escore do GI na prova de compreensão de leitura de sentenças e de consciência fonológica. O mesmo grupo apresentou diminuição significativa no tempo de execução de provas de leitura e de escrita de palavras isoladas. Nesse sentido, a intervenção trouxe benefícios para a leitura dos disléxicos.
\end{abstract}

Palavras-chave: Dislexia, Intervenção, Leitura, Escrita.

\section{Assessment of phonics-based literacy software for children with developmental dyslexia}

\begin{abstract}
The Phonological Deficit theory explains confusions and deficits in phonological awareness presents in subjects with Developmental Dyslexia. Interventions focused on this process contributes to improvement in reading. The study examined the effectiveness of a Phonic Literacy Software in promoting phonological awareness and graphophonemic correspondences in dyslexic. Twenty dislexic children were divided in two groups (GI and GII) and were paired by age, sex and schooling. All subjects performed tests of phonological awareness and reading skills and writing. The participants of GI participated in 16 intervention sessions and, after this period, the groups were reevaluated. There was significant increase in the score of the GI in reading comprehension of sentences task and phonological awareness task. The same group presented significant decrease in execution time in word reading and written words tasks. Therefore, the intervention brought benefits for reading of dyslexics.

Keywords: Dyslexia, Early intervention, Reading, Writing.
\end{abstract}

Uma vez diagnosticado um quadro de distúrbio específico de aprendizagem da leitura e da escrita, a busca por programas de tratamento adequados torna-se um desafio aos profissionais que trabalham diretamente com essas crianças (Bermejo \& Escribano, 2005; Capovilla \& Capovilla, 2000; Torgesen, 2001). Para esses casos é importante considerar o uso de programas de intervenção estruturados e de eficácia comprovada para a remediação de dificuldades da linguagem escrita, critérios, estes, que vêm sendo adotados em diversos países, tais como EUA, França e Reino Unido (Smythe, Everatt \& Salter, 2004).

A seleção das atividades que devem constar em tais programas se dá em função do conhecimento sobre a aquisição da linguagem escrita, seus componentes e os possíveis distúrbios neste processo, ou seja, deve-se considerar que a leitura envolve diversos processos cognitivos que ocorrem

1 Endereço para correspondência:

Rua Piauí, n $181,10^{\circ}$ andar - 01241-000 - São Paulo - SP

.E-mail: elizeumacedo@uol.com.br. simultaneamente, tais como: percepção visual, movimento ocular, associação visuoauditiva, reconhecimento auditivo, processamento fonológico, memória visual, memória auditiva, expressão oral e processos verbais superiores (Etchepareborda, 2003). Além disso, as habilidades de consciência fonológica, que se referem ao reconhecimento e à manipulação dos componentes das palavras, das sílabas ou dos fonemas, desempenham papel importante para a aquisição da leitura no sistema alfabético do português (Guimarães, 2003; Cardoso-Martins, 2008).

Considerando que o processo de aquisição das habilidades de leitura e de escrita é gradual, o modelo proposto por Frith (1990) descreve três fases, ou estratégias, que ocorrem na seguinte ordem: logográfica, alfabética e ortográfica. Na leitura e na escrita logográfica as palavras são percebidas como desenhos e o leitor usa pistas contextuais. $\mathrm{Na}$ fase alfabética dá-se início ao processo de acesso à representação fonológica das palavras, com isolamento das unidades fonéticas individuais e mapeamento dos mesmos nas letras correspondentes. $\mathrm{Na}$ última fase ocorre a leitura e a escrita ortográfica, na qual as crianças leem e escrevem reconhecendo as unidades 
morfêmicas das palavras e está relacionada diretamente ao sistema semântico e grafêmico lexical. De acordo com esse modelo, interrupções na progressão de uma a outra fase ao longo do desenvolvimento da leitura podem levar a distúrbios de leitura. Tal interrupção pode ocorrer já na passagem da fase logográfica para a alfabética, ou apenas na passagem da fase alfabética para a ortográfica.

A dislexia do desenvolvimento é o mais comum e estudado dos transtornos de aprendizagem, sendo sua origem neurobiológica (Ciasca, Capellini \& Tonelotto, 2003; Galaburda \& Cestnick, 2003; Shastry, 2007), e de acordo com o DSM-IV-TR (American Psychiatric Association, 2003), os critérios para diagnóstico incluem as aptidões para a leitura significativamente inferiores ao nível esperado, considerando-se a escolarização do indivíduo e suas capacidades intelectuais; interferência significativa no desempenho escolar ou atividades diárias em razão das perturbações de leitura e ausência de disfunção visual, auditiva ou neurológica. O caráter neurobiológico e constitucional da dislexia é confirmado por achados recorrentes de estudos que apontam padrões de herdabilidade de dislexia em famílias; padrões diferenciados de ativação cerebral observados com registro de neuroimagem, como hipoativação do lobo esquerdo em tarefas linguísticas; e, por fim, diferenças no processamento visual e padrão de movimentos oculares (Fletcher, 2009).

A avaliação para preenchimento dos critérios diagnósticos, na maioria das vezes, é baseada nos sintomas observáveis do comportamento da leitura e da escrita. Nesse sentido, a adoção de instrumentos e procedimentos de avaliação dos diferentes aspectos da leitura e da escrita é essencial a fim de identificar o nível de desenvolvimento da leitura, bem como a qualidade dos prejuízos apresentados pelo indivíduo. Além disso, a compreensão e análise dos tipos de erro, quando realizadas à luz de modelos cognitivos de leitura e de escrita, permitem verificar o subtipo da dificuldade de leitura, que compreende dois perfis neuropsicológicos mais frequentes, o subtipo fonológico, em que ocorre uma espécie de interrupção da fase logográfica para a alfabética, e o morfêmico, em que há dificuldades na aquisição das representações ortográficas (Frith, 1990). Em ambos os casos há dificuldades no reconhecimento de palavras, apesar de a compreensão de textos ou de informações não ser prejudicada quando transmitidas oralmente (Capovilla \& Capovilla, 2002). Neste sentido, os programas interventivos devem considerar as particularidades de déficits encontrados na dislexia do desenvolvimento. Os estudos mais recentes têm verificado os alcances e limitações de diferentes procedimentos e programas (Wanzek \& Vaughn, 2008).

Odegard, Ring, Smith, Biggan e Black (2008) conduziram um experimento para verificar a eficácia de um procedimento interventivo sobre o padrão de ativação cerebral em sujeitos disléxicos e saudáveis. A intervenção contemplou cinco componentes: consciência fonológica, correspondência grafemafonema, vocabulário, fluência de leitura e compreensão de pequenas frases e de textos. Para trabalhar os componentes, $35 \%$ do tempo de cada sessão foi dedicado às instruções de habilidades fonológicas, sendo $25 \%$ para fonemas e $10 \%$ para consciência fonológica. Outros $30 \%$ do tempo foram usados para treino de compreensão de textos e aumento de vocabulário, sendo $20 \%$ e $10 \%$, respectivamente. Os exercícios para fluência na leitura ocupavam 18\% do tempo de instrução e o tempo restante foi usado nas instruções faladas. Os resultados mostraram que a ativação no lobo parietal inferior esquerdo dos respondentes à intervenção foi diferenciada do padrão de crianças não respondentes, aquelas com dificuldades persistentes na leitura de palavras isoladas, e controles.

Gustafson, Ferreira e Ronnberg (2007) verificaram a eficácia de dois procedimentos de intervenção destinados a crianças com dificuldades específicas fonológicas e ortográficas, tendo sido cada grupo submetido à intervenção mais adequada ao perfil de dificuldade apresentada. O programa fonológico foi composto por atividades de rima, de adição, de segmentação e de inversão fonêmica. Já o treino ortográfico incluiu sessões de leitura de palavras e de textos, de memorização de morfemas e de construção de palavras. As avaliações pós-intervenção não mostraram diferenças significativas no desempenho entre grupos e indicam que ambos os treinos promoveram desenvolvimento da leitura e da consciência fonológica.

Em estudo conduzido por Rashotte, MacPhee e Torgesen (2001) foi aplicado um programa de intervenção em leitura em grupos de três a cinco escolares com baixos níveis de habilidades de decodificação fonêmica e de leitura, selecionados de escolas públicas. O grupo experimental (GE) recebeu tratamento durante oito semanas, enquanto o grupo controle (GC) recebeu apenas as instruções de leitura comuns na sala de aula. No primeiro pós-teste, o GE teve desempenho significativamente melhor que o GC em provas de consciência e de decodificação fonológica, de precisão na leitura, de compreensão e de ortografia. Os resultados do segundo pós-teste, feito após o GC também se submeter ao programa, indicam padrão similar no desenvolvimento da habilidade de leitura. 
Torgesen (2001) comparou dois métodos de intervenção em crianças com dificuldades de leitura, distribuídas em dois grupos, que receberam 67,5 horas de intervenção intensiva cada. As sessões eram diárias, em duas etapas de 50 minutos, cinco dias por semana, durante oito semanas. Em um dos grupos, as crianças receberam o programa LIPS (Programa Sequencial Lindamood para Leitura e Escrita), cujo objetivo era fortalecer as habilidades de consciência fonológica por meio do treino fonoarticulatório. $\mathrm{O}$ outro grupo foi submetido ao programa EP (Fortalecimento Fônico), que estimulava as crianças a escreverem palavras já aprendidas e a ouvirem os sons na palavra como auxílio à ortografia. Também foram lidos textos com o objetivo de proporcionar generalização das regras de decodificação para a leitura a partir da identificação dos erros cometidos, os quais foram cuidadosamente corrigidos pelos professores.

Ambos os programas descritos consideraram ensino explícito e intensivo de estratégias de decodificação fonêmica e sugestão de estratégias adequadas para a leitura de palavras ou texto. No entanto, os programas diferiram quanto ao tempo dedicado às diferentes atividades: para o treino de consciência fonológica e de decodificação fonêmica, o LIPS usou $85 \%$ do tempo da intervenção e o EP usou $20 \%$ do tempo; para a escrita de palavras isoladas, o tempo gasto foi de $10 \%$ para o LIPS e de $30 \%$ para o EP; por fim, o tempo utilizado para a leitura e a escrita de textos foi de 5\% para o LIPS e de $50 \%$ para o EP. O desempenho das crianças na avaliação pósintervenção revelou que os participantes do treino com o programa LIPS obtiveram melhores resultados na decodificação fonológica de palavras e textos, e os participantes do EP conseguiram melhor execução na compreensão dos textos lidos.

Em estudo de Salgado e Capellini (2008), 24 crianças brasileiras foram classificadas em 4 grupos: GI, subdividido em GIe (disléxicos submetidos à intervenção fonológica), GIc (disléxicos não submetidos ao programa de intervenção), GII, subdivido em GIIe (escolares bons leitores submetidos à intervenção) e GIIc (escolares bons leitores não submetidos à intervenção). Após a intervenção fonológica, o grupo GIe apresentou desempenho semelhante ao GII, evidenciando a eficácia do programa. O programa foi realizado em 20 sessões de 40 minutos. O único grupo que apresentou evolução no estágio de leitura foi o GIe e, em ambos os grupos submetidos à intervenção, a velocidade de leitura aumentou. Esses dados, segundo os autores, revelam a importância da implantação de programas com foco nas habilidades fonológicas para posterior desenvolvimento da automatização no acesso lexical, do mecanismo de conversão grafofonêmico e da compreensão da leitura.

Em artigo de revisão sobre métodos de tratamento da dislexia, Shaywitz, Morris e Shaywitz (2008) colocam que os métodos que enfatizam o desenvolvimento do principio alfabético por meio do ensino das correspondências grafema-fonema, tais como os fônicos, são os que alcançam melhores resultados em comparação aos métodos globais, pois se caracterizam por serem estruturados, sequenciais, acumulativos, sistemáticos e multissensoriais. Os estudos de intervenção em leitura, como esse, podem beneficiar tanto escolas quanto psicopedagogos e educadores, fornecendo subsídios científicos acerca dos programas que poderão ser mais bem utilizados e terão maior alcance entre os estudantes, com o menor custo e maior aproveitamento (Rashotte, MacPhee \& Torgesen, 2001). Sendo assim, o objetivo desta pesquisa foi verificar a eficácia da intervenção com o Software Alfabetização Fônica Computadorizada nas habilidades de consciência fonológica e de correspondências grafofonêmicas em crianças com dislexia do desenvolvimento.

\section{Método}

\section{Participantes}

Vinte participantes com diagnóstico de dislexia do desenvolvimento, com idade média de 11,7 anos, foram alocados em dois grupos. O primeiro grupo (GI) foi formado por disléxicos submetidos à intervenção com o Software Alfabetização Fônica Computadorizada. O segundo grupo (GII) foi composto pelos disléxicos que não participaram do programa de intervenção com o software, a fim de se caracterizar como grupo controle. O GI foi formado por 10 crianças $(7$ meninos) com idade média de 11 anos (2,44 dp) e 4,6 anos de escolaridade. Já o GII foi formado por 10 crianças (7 meninos) com idade média de 10,8 anos (1,75 dp) e 4,9 anos de escolaridade. Os critérios de pareamento para a formação dos grupos foram idade, sexo e a série escolar.

Além disso, foi considerado para a seleção dos participantes o padrão de leitura apresentado pelos disléxicos, sendo escolhidos os que obtiveram perfil compatível com o subtipo de dislexia fonológica, ou seja, leitura fonológica significativamente inferior. Para isso utilizou-se o resultado obtido no Teste de Competência de Leitura de Palavras e Pseudopalavras (TCLPP), uma vez que o tipo de erro cometido revela a estratégia de leitura utilizada e a estratégia na qual o participante apresenta dificuldade (Capovilla e colaboradores, 2007). Para a divisão dos participantes no GI e no GII foi adotado um critério de 
conveniência, alocando os 10 primeiros participantes selecionados no grupo de intervenção.

Os participantes foram diagnosticados e encaminhados para a participação na pesquisa pelo Núcleo de Atendimento Neuropsicológico Infantil Interdisciplinar (NANI-UNIFESP) e por consultórios particulares de atendimento fonoaudiológico. Todos foram avaliados por meio de uma bateria computadorizada de leitura, de escrita e de consciência fonológica, a fim de satisfazer os critérios de inclusão e exclusão.

Os critérios de exclusão adotados no estudo foram: 1) QI Total no WISC III abaixo da média, com percentil geral abaixo de 25 ; 2) suspeita de deficiências visuais e/ou auditivas sem correção; 3) presença de lesões cerebrais, histórico de doenças clínicas neurológicas ou psiquiátricas; 4) atraso no desenvolvimento de linguagem oral ou atraso neuropsicomotor; 5) a presença de comorbidade com o transtorno déficit de atenção e hiperatividade.

\section{Instrumentos}

Os instrumentos de avaliação foram uma Prova de Consciência Fonológica Computadorizada e cinco testes da BALE Computadorizada (Macedo e colaboradores, 2005): o Teste de Competência de Leitura de Sentenças (TCLS), que avalia a capacidade de leitura e compreensão de sentenças; o Teste de Compreensão de Sentenças Faladas (TCSF), que avalia a compreensão de sentenças faladas; o Teste de Nomeação de Figuras por Escrita (TNF 1.1-Escrita), que verifica a habilidade de escrita de palavras por meio da nomeação de figuras; o Teste de Nomeação de Figuras por Escolha (TNF 1.1-Escolha), que indica a competência na escolha de palavras pela leitura; e o Teste de Competência de Leitura de Palavras e Pseudopalavras (TCLPP), que avalia o julgamento ortográfico de diferentes categorias de palavras. O teste é composto de 70 pares figura-palavra, e a criança deve julgar a adequação dos pares. As 7 categorias avaliadas são: CR - corretas regulares (ex: pipa sob a figura de uma pipa), CI - corretas irregulares (ex: táxi sob a figura de um táxi), VS - palavras vizinhas semânticas (ex: laranja sob a figura de uma banana), PE pseudopalavras estranhas (ex: rassuno sob a figura de uma mão), PH - pseudopalavras homófonas (ex: páçaru sob a figura de um pássaro), TF pseudopalavras com trocas fonológicas (ex: relóchio sob a figura de um relógio), e por fim, TV pseudopalavras com trocas visuais (ex: esterla sobre a figura de uma estrela). Além disso, o teste permite interpretar o padrão de leitura específico de uma criança segundo modelo cognitivo de desenvolvimento de leitura e de escrita, e inferir o estágio de desenvolvimento de acordo com as fases propostas por Frith (Capovilla, Varanda \& Capovilla, 2006). O estudo de Diana (2007) mostrou que a BALE Computadorizada apresenta validade de critério, pois o desempenho no teste variou em função da idade e da série escolar. No entanto, ainda não foram realizados estudos de fidedignidade para a versão computadorizada dos testes.

O instrumento de intervenção foi o Software Alfabetização Fônica Computadorizada (Capovilla, Capovilla, Macedo \& Diana, 2005), cujas atividades estimulam ludicamente as habilidades de manipulação de grafemas e fonemas. O objetivo dessas atividades é promover a aquisição da leitura alfabética, que ocorre por meio da decodificação grafema-fonema. O software é dividido em dois módulos de atividades: Consciência Fonológica e Alfabeto. O módulo Consciência Fonológica contém atividades de reconhecimento de palavras, rima, aliteração, sílabas e fonemas, sendo os estímulos apresentados em desenhos ou em formas geométricas. $\mathrm{Na}$ atividade palavras, o participante deve identificar palavras dentro de uma frase e inserir palavras em frases incompletas mediante combinação semântica. Nas atividades rima, aliteração, sílabas e fonemas, os itens são apresentados inicialmente por meio de figuras que devem ser combinadas de acordo com o princípio fonético do nome e, em seguida, as unidades sonoras são apresentadas em formatos geométricos, para a realização de operações de adição, subtração ou inversão das unidades sonoras.

Já o módulo Alfabeto é subdividido nas seções vogais e consoantes. Para cada grafema é apresentado o fonema correspondente, que é ouvido pela criança quando ela faz o contorno da letra com o mouse. Em seguida são apresentadas listas de palavras para leitura e exercícios de discriminação fonológica para identificação de sons que completam as palavras.

\section{Procedimentos}

A pesquisa foi aprovada pelo Comitê de Ética da UPM sob protocolo número 1087/08. Os responsáveis por todos os participantes assinaram o Termo de Consentimento Livre e Esclarecido e estes foram avaliados no Laboratório de Neurociência Cognitiva da Universidade Presbiteriana Mackenzie. De acordo com os princípios éticos, após a análise dos resultados do estudo, os participantes do grupo sem intervenção também foram submetidos aos procedimentos, uma vez que estes trouxeram benefícios nas habilidades de leitura e escrita.

Após a avaliação de todos os participantes no pré-teste, os procedimentos foram diferentes para os dois grupos. Assim, o GI foi submetido à intervenção com sessões semanais e individuais de 
aproximadamente 60 minutos de duração. A ordem de execução das atividades utilizada foi a prevista pelo manual do software, que intercala atividades dos módulos Consciência Fonológica e Alfabeto, com aumento crescente de dificuldade das atividades. O número de sessões variou conforme a realização do protocolo do software, e cada participante executou duas vezes todas as atividade propostas, o que totalizou um número médio de dezesseis sessões de intervenção. Já os participantes do GII não participaram de nenhuma atividade que envolvesse $\mathrm{O}$ treino de habilidades fônicas e continuaram frequentando o ensino regular. A fim de verificar o efeito da intervenção e controlar possíveis fontes de invalidação interna (Campbell \& Stanley, 1963), os participantes dos dois grupos foram submetidos novamente à avaliação. Dessa forma foi possível comparar o desempenho entre os grupos, bem como entre o pré e pós-teste, pois foram utilizados os mesmos instrumentos nas duas avaliações.

\section{Resultados}

Para comparar o nível de leitura e de escrita dos grupos antes da intervenção e verificar se ambos apresentavam desempenhos homogêneos nas tarefas, foi conduzida comparação das médias entre o GI e o GII e ANOVA de medidas repetidas. Não foram encontradas diferenças significativas entre os desempenhos dos grupos na avaliação pré-teste. A Tabela 1 ilustra os resultados do GI e do GII antes da intervenção.

Tabela 1 - Média de número de acertos e tempo de execução em cada item (em segundos) no pré-teste - Teste de Competência de Leitura de Palavras e Pseudopalavras (TCLPP), Teste de Compreensão de Leitura de Sentenças (TCLS), Teste de Compreensão de Sentenças Faladas (TCSF), Teste de Nomeação de Figura por Escrita (TNF 1.1 - Escrita), Teste de Leitura por Escolha de Palavras (TNF 1.1 - Escolha) e Prova de Consciência Fonológica (PCF)

\begin{tabular}{|c|c|c|c|c|c|c|}
\hline & & Grupo & $\mathrm{N}$ & Média (dp) & $\mathrm{F}(1,15)$ & $P$ \\
\hline \multirow{4}{*}{ TCLPP } & Acertos & GI & 10 & $57,60( \pm 6,484)$ & & \\
\hline & & GII & 10 & $58,10( \pm 6,903)$ & 0,028 & 0,869 \\
\hline & Tempo & GI & 10 & $10,096( \pm 7,962)$ & & \\
\hline & & GII & 10 & $8,182( \pm 6,398)$ & 0,10 & 0,922 \\
\hline \multirow{4}{*}{ TCLS } & Acertos & GI & 8 & $32( \pm 10,254)$ & & \\
\hline & & GII & 9 & $32,78( \pm 13,283)$ & 0,018 & 0,895 \\
\hline & Tempo & GI & 8 & $15,497( \pm 10,704)$ & & \\
\hline & & GII & 9 & $14,346( \pm 2,401)$ & 0,888 & 0,771 \\
\hline \multirow{4}{*}{ TCSF } & Acertos & GI & 10 & $38,10( \pm 3,573)$ & & \\
\hline & & GII & 10 & $38,7( \pm 6,343)$ & 0,068 & 0,797 \\
\hline & Tempo & GI & 10 & $8,176( \pm 1,395)$ & & \\
\hline & & GII & 10 & $6,778( \pm 1,707)$ & 4,015 & 0,060 \\
\hline \multirow{4}{*}{ TNF 1.1-Escrita } & Acertos & GI & 10 & $13,60( \pm 7,336)$ & & \\
\hline & & GII & 10 & $20,30( \pm 8,667)$ & 3,481 & 0,078 \\
\hline & Tempo & GI & 10 & $25,90( \pm 13,852)$ & & \\
\hline & & GII & 10 & $19,27( \pm 12,158)$ & 1,293 & 0,270 \\
\hline \multirow{4}{*}{ TNF 1.1-Escolha } & Acertos & GI & 10 & $29,10( \pm 3,281)$ & & \\
\hline & & GII & 10 & $30,60( \pm 6,222)$ & 0,455 & 0,509 \\
\hline & Tempo & GI & 10 & $7,53( \pm 3,744)$ & & \\
\hline & & GII & 10 & $6,49( \pm 5,611)$ & 0,238 & 0,631 \\
\hline \multirow{4}{*}{ PCF } & Acertos & GI & 9 & $40,22( \pm 14,087)$ & & \\
\hline & & GII & 10 & $46,20( \pm 13,571)$ & 0,887 & 0,360 \\
\hline & Tempo & GI & 9 & $20,70( \pm 8,773)$ & & \\
\hline & & GII & 10 & $24,02( \pm 8,064)$ & 0,791 & 0,386 \\
\hline
\end{tabular}

Para verificar o efeito da intervenção por meio da comparação entre os desempenhos dos grupos, foi calculada a diferença entre as pontuações e tempos de execução do pós e pré-teste. A Tabela 2 mostra as médias das diferenças de pontuação e de tempo de execução da condição pós para a condição pré-teste no GI e GII. 
Tabela 2 - Média de diferença entre acertos e tempo de execução em cada item (em segundos) nos acertos do pós para o préteste - Teste de Competência de Leitura de Palavras e Pseudpalavras (TCLPP), Teste de Compreensão de Leitura de Sentenças (TCLS), Teste de Compreensão de Sentenças Faladas (TCSF), Teste de Nomeação de Figura por Escrita (TNF 1.1 - Escrita), Teste de Leitura por Escolha de Palavras (TNF 1.1 - Escolha) e Prova de Consciência Fonológica (PCF)

\begin{tabular}{|c|c|c|c|c|c|c|}
\hline & & Grupo & $\mathrm{N}$ & Média (dp) & $\mathrm{F}(1,15)$ & $P$ \\
\hline \multirow{4}{*}{ TCLPP } & Acertos & GI & 10 & $2,40( \pm 6,114)$ & \multirow{3}{*}{0,341} & \multirow{3}{*}{0,566} \\
\hline & & GII & 10 & $1,20( \pm 2,201)$ & & \\
\hline & Tempo & GI & 10 & $-2,18( \pm 6,475)$ & & \\
\hline & & GII & 10 & $1,31( \pm 0,324)$ & 5,224 & 0,035 \\
\hline \multirow{4}{*}{ TCLS } & Acertos & GI & 8 & $6,30( \pm 5,143)$ & \multirow{2}{*}{13,742} & \multirow{2}{*}{0,002} \\
\hline & & GII & 9 & $0,20( \pm 0,789)$ & & \\
\hline & Tempo & GI & 8 & $8,44( \pm 17,466)$ & & \\
\hline & & GII & 9 & $-0,22( \pm 0,736)$ & 2,460 & 0,134 \\
\hline \multirow{4}{*}{ TCSF } & Acertos & GI & 10 & $3,50( \pm 4,503)$ & \multirow{2}{*}{1,349} & \multirow{2}{*}{0,261} \\
\hline & & GII & 10 & $1,60( \pm 2,547)$ & & \\
\hline & Tempo & GI & 10 & $-1,94( \pm 13,518)$ & & \\
\hline & & GII & 10 & $0,11( \pm 0,412)$ & 21,399 & 0,000 \\
\hline \multirow{4}{*}{ TNF 1.1 - Escrita } & Acertos & GI & 10 & $2,20( \pm 3,853)$ & \multirow{3}{*}{2,160} & \multirow{2}{*}{0,159} \\
\hline & & GII & 10 & $-0,90( \pm 5,446)$ & & \\
\hline & Tempo & GI & 10 & $-5,47( \pm 8,281)$ & & \\
\hline & & GII & 10 & $3,78( \pm 9,495)$ & 5,406 & 0,032 \\
\hline \multirow{4}{*}{ TNF 1.1 - Escolha } & Acertos & GI & 10 & $3,40( \pm 2,797)$ & \multirow{2}{*}{2,133} & \multirow{3}{*}{0,161} \\
\hline & & GII & 10 & $1,80( \pm 2,044)$ & & \\
\hline & Tempo & GI & 10 & $-1,86( \pm 2,843)$ & & \\
\hline & & GII & 10 & $0,11( \pm 1,575)$ & 5,054 & 0,037 \\
\hline \multirow{4}{*}{ PCF } & Acertos & GI & 9 & $11,20( \pm 10,581)$ & \multirow{3}{*}{9,837} & \multirow{3}{*}{0,006} \\
\hline & & GII & 10 & $-3,30( \pm 10,089)$ & & \\
\hline & Tempo & GI & 9 & $3,71( \pm 9,804)$ & & \\
\hline & & GII & 10 & $-1,24( \pm 2,966)$ & 2,351 & 0,143 \\
\hline
\end{tabular}

ANOVAS de medidas repetidas foram conduzidas a fim de verificar diferenças entre os dois grupos, após a intervenção para o número de acertos e tempo de execução dos testes. Análises indicam aumento significativo do número de itens corretos do GI em comparação ao GII no teste TCLS e na PCF. Assim, no TCLS foi observada uma diferença significativa entre os dois grupos, sendo que os participantes do GI acertaram em média 6 itens a mais do que os do GII $(F[1,15]=13,742 ; p=0,002)$. Já na PCF, os participantes do GI acertaram, em média, 14 itens a mais do que os participantes do GII $(F[1,15]=9,837 ; \quad p=0,006)$. Não foram observadas diferenças na pontuação para os demais testes. Análise do tempo para realização dos testes revelou que os participantes do GI foram mais rápidos do que o grupo controle em XXX testes. Assim, no TCLPP, os participantes do GI levaram, em média, 2 segundos a menos do que os participantes do GII para responder cada item $(F[1,15]=5,224 ; p=0,035)$. Padrão similar foi encontrado no TCSF, pois os participantes do GI foram significativamente mais rápidos do que os do GII $(F[1,15]=21,399 ; \mathrm{p}=0,000)$. No TNF 1.1 - Escrita, os participantes do GI levaram, em média, 8 segundos a menos para escrever cada item $(\mathrm{F}[1,15]=5,406$, $\mathrm{p}=0,032)$. Por fim, no TNF 1.1 - Escolha foi observada redução de cerca de 2 segundos para julgamento e escolha de cada item $(\mathrm{F}[1,15]=5,054$, $\mathrm{p}=0,037)$. Verificou-se ainda que, nas provas em que não houve diferença no tempo de execução, também não foram observadas diferenças no número de itens respondidos corretamente, o TCLS $(\mathrm{F}[1,15]=8,448$; $\mathrm{p}=0,134)$ e a $\operatorname{PCF}(\mathrm{F}[1,15]=3,717 ; \mathrm{p}=0,143)$.

Embora não tenha sido encontrada diferença significante entre os dois grupos para número total de acertos e tempo de realização do TCLPP, ANOVAs de medidas repetidas foram conduzidas para verificar possíveis efeitos do treino para as 7 categorias desse teste. Resultados mostram que não houve diferença significativa na pontuação entre os dois grupos. No entanto, observa-se uma mudança no padrão de acerto entre os dois grupos para cada uma das categorias. Assim, para os participantes do GII, o grau de complexidade das categorias variou das mais fáceis para as mais difíceis, na seguinte ordem: PE (pseudopalavras estranhas) $=\mathrm{CR} \quad$ (Corretas regulares) $=\mathrm{VS} \quad$ (vizinhas 
semânticas) $>$ CI (corretas irregulares) $>$ TV (pseudopalavras com troca visual) $>$ TF (pseudopalavras com trocas fonológicas) $>\mathrm{PH}$ (pseudopalavras homófonas). Tais resultados mostram não haver diferença significativa entre os seguintes itens: pseudopalavras estranhas, corretas regulares e vizinhas semânticas e, além disso, a categoria de pseudopalavras homófonas foi a mais difícil. Já para os participantes do GI, o grau de dificuldade das categorias mais fáceis para as mais difíceis foi a seguinte: $\mathrm{PE}>\mathrm{VS}>\mathrm{CI}>\mathrm{CR}>\mathrm{TV}>\mathrm{TF}>\mathrm{PH}$. Dessa forma, os participantes do GI apresentaram padrão diferente de resposta entre todas as categorias e, como no GII, a categoria $\mathrm{PH}$ foi aquela em que os participantes mais encontraram dificuldades de julgamento. Além disso, as categorias de palavras com maiores aumentos de pontuação do pré para o pós-teste dos participantes do GI foram: CR, CI, PH e TV.

\section{Discussão}

Resultados revelam aumento significativo na pontuação do GI no teste que avalia habilidades de consciência fonológica (PCF) e de compreensão de sentenças escritas (TCLS). Tais resultados indicam que o programa interventivo utilizado pode ser eficaz no desenvolvimento de habilidades metalinguísticas necessárias à leitura competente, bem como na capacidade de compreensão de frases e textos. O aumento na taxa de acerto no TCLS não foi acompanhado de aumento no tempo de realização do teste. $\mathrm{O}$ aumento na taxa de acerto, sem aumento do tempo de realização nesse teste, pode ser decorrente do tipo de intervenção feita no GI, uma vez que o ensino sistemático das correspondências grafofonêmicas possibilita a automatização da decodificação da leitura. Embora tenha sido observado aumento na capacidade de automatização dessas crianças e adolescentes, ainda estão abaixo do que seria esperado para a idade e série escolar. Ou seja, apesar do aumento na taxa de acertos, os participantes do GI ainda levam mais tempo do que os leitores normais para realizar as provas com precisão e fluência (Lukasova, Oliveira, Barbosa \& Macedo, 2008).

Os sujeitos que participaram da intervenção apresentaram diminuição significativa do tempo de execução de provas que avaliam tanto a leitura quanto a escrita de palavras isoladas. Tais resultados são similares aos de outros estudos (Salgado \& Capellini, 2008), que mostram que a melhora na capacidade de decodificação promove aumento na velocidade de leitura, por meio da facilitação de acesso ao léxico ortográfico (Boder, 1973; Share, 1995). De fato, estudos indicam que crianças com dislexia fazem predominantemente leitura visual global, conforme o esperado na fase logográfica de desenvolvimento da leitura (Capovilla \& Capovilla, 2000; Frith, 1990). Nesses casos, o acesso à representação de palavras é feito sem o uso competente das estratégias alfabéticas e ortográficas em virtude das dificuldades no processamento fonológico.

Em relação às habilidades de decodificação grafema-fonema, que foram treinadas no programa de intervenção, estas parecem fortalecer $O$ desenvolvimento da estratégia alfabética de leitura (Frith, 1990). Esse desenvolvimento pode explicar o aumento do número de acertos em algumas categorias do TCLPP, tais como as palavras corretas regulares e pseudopalavras com trocas visuais. No entanto, como esses aumentos não foram significativos para todas as categorias, estudos que treinem especificamente habilidades ortográficas podem revelar ganhos maiores em provas como o TCLPP (Fletcher, 2009). De fato, estudos que integrem o treino de habilidades ortográficas com habilidades fonológicas podem produzir efeito maior no desempenho de pessoas com dislexia do desenvolvimento em provas de leitura e escrita (Torgesen, 2001).

Embora o programa de intervenção tenha produzido mudança de desempenho do GI em alguns testes, tal padrão não foi observado em todos os testes. Assim, não foram encontradas diferenças significativas no número de acertos entre os dois grupos de participantes nos seguintes testes: TCLPP, TCSF, TNF 1.1 - Escrita e TNF 1.1 - Escolha. Esses dados podem ser analisados em função de fatores constitucionais da dislexia do desenvolvimento. Ou seja, Odegard \& colaboradores (2008) mostram, em outro estudo, interventivo, que alguns participantes disléxicos não foram respondentes ao tratamento e não apresentaram melhora significativa para leitura de palavras isoladas. Esse fato pode explicar a persistência encontrada no padrão de erros no TCLPP, nas categorias pseudopalavras homófonas e pseudopalavras com troca fonológica.

É importante ressaltar que procedimentos de intervenção similar ao deste estudo, conduzido com crianças da $1^{\mathrm{a}}$ e $2^{\mathrm{a}}$ séries do Ensino Fundamental de escolas regulares, revelou padrão diferente de resposta. Nikaedo (2007) observou que crianças do grupo experimental, e que foram submetidas à programa de intervenção fônica, tiveram taxa de acertos maior do que as controles em todas as categorias do TCLPP, exceto em pseudopalavras homófonas. Diferente do presente estudo, Nikaedo (2007) realizou trabalhou com crianças que tinham desenvolvimento cognitivo normal para leitura. No entanto, a falta de efeito em estudos com disléxicos parece estar relacionada com o 
fato de crianças disléxicas apresentarem traços característicos de dificuldades no processamento visual e fonológico de palavras. Esses traços tendem a permanecer ao longo do tempo (Rashotte, MacPhee \& Torgesen, 2001). Assim, para que efeitos mais robustos possam ser identificados, faz-se necessária a condução de novos estudos em que o intervalo entre as sessões, bem como o número de encontros, sejam maiores do que o do presente estudo.

Neste foi adotado o modelo de intervenção clínico e individual, com intervalo de tempo entre as sessões de uma semana. Outros estudos de intervenção com disléxicos utilizam intervalos menores entre as sessões e número maior delas, o que pode explicar as maiores diferenças observadas entre os grupos, gerando resultados mais robustos (Rashotte, MacPhee \& Torgesen, 2001; Odegard e colaboradores, 2008). Assim, a realização de novos estudos brasileiros com intervalo menor entre as sessões e número maior de encontros responderá se o tipo de procedimento adotado pode ser mais eficaz.

Com base nos resultados obtidos neste estudo, sugere-se a realização de novos procedimentos de intervenção com instrumentos que incluam atividades de favoreçam o ensino de habilidades ortográficas. Além disso, a análise dos resultados obtidos por intervenções feitas por professores, em vez do pesquisador, pode revelar aspectos relacionados com a validade ecológica deste tipo de procedimento. Outro caminho a ser explorado é a criação e adequação do software para outros grupos ou contextos interventivos, a fim de analisar o efeito diferencial em diferentes faixas etárias e condições: intervenção precoce em préescolares, adolescentes e adultos com dificuldades de leitura. Por fim, outra perspectiva de estudos envolve, por exemplo, a comparação dos resultados de intervenções computadorizadas com a feita por professores em sala de aula, com uso de outros materiais didáticos.

Conclui-se, portanto, que a utilização do Software Alfabetização Fônica Computadorizada promoveu melhora significativa nas habilidades de leitura e compreensão de sentenças escritas e de consciência fonológica. Além disso, interferiu significativamente na velocidade de leitura e escrita de palavras isoladas. Sendo assim, o uso deste instrumento pode ser eficaz no desenvolvimento de competência para leitura e escrita de crianças com dislexia do desenvolvimento.

\section{Referências}

American Psychiatric Association. (2003). DSM-IV-TR: Manual diagnóstico e estatístico de transtornos mentais. $\left(4^{\mathrm{a}}\right.$ Ed.). Porto Alegre: Artmed.

Bermejo, V. S. \& Escribano, C. L. (2005). Nuevos aportes a la intervención en las dificuldades de lectura. Universitas Psychologica, 4(11), 13-22.

Boder, E. (1973). Development dyslexia: a diagnostic approach based on three atypical reading-spelling patterns. Development medicine and child neurology, 15 (2), 663-687.

Campbell, D. T. \& Stanley, J. C. (1963). Experimental and quasi-experimental designs for research. Boston: Houghton Mifflin.

Capovilla, A. G. S. \& Capovilla, F. C. (2000). Problemas de leitura e escrita: como identificar remediar e prevenir numa abordagem fônica. São Paulo: Memnon.

Capovilla, A. G. S. \& Capovilla, F. C. (2002). Alfabetização: método fônico. São Paulo: Memnon.

Capovilla, A. G. S., Capovilla, F. C., Macedo, E. \& Diana, C. A. (2005). Alfabetização fônica computadorizada: CD-ROM. São Paulo: Memnom.

Capovilla, F. C., Varanda, C. \& Capovilla, A. G. S. (2006). Teste de competência de leitura de palavras e pseudopalavras: normatização e validação. Revista de Psicologia da Vetor Editora, 7 (2), 47-59.

Capovilla, A. G. S., Dias, N. M., Trevisan, B. T., Capovilla, F. C., Rezende, M. C. A., Andery, M. A. \& Lopes, F. (2007). Avaliação de leitura em crianças disléxicas: teste de competência de leitura de palavras e pseudopalavras. Em: A. G. S. Capovilla, \& F. C. Capovilla (Orgs.). Teoria e pesquisa em avaliação neuropsicológica. (pp. 36-44). São Paulo: Memnon.

Cardoso-Martins, C. (2008). Desenvolvimento das habilidades de leitura e escrita. Em: D. Fuentes, L. F. Malloy-Diniz, C. H. P. Camargo \& R. M. Cosenzal. Neuropsicologia: teoria e prática. Porto Alegre: Artmed.

Ciasca, S. M., Capellini, A. S. \& Tonelotto, J. M. F. (2003). Distúrbios específicos de aprendizagem. Em: S. M. Ciasca (Org.). Distúrbios de Aprendizagem: Proposta de Avaliação Interdisciplinar. (pp. 19-45). São Paulo: Casa do Psicólogo. 
Diana, C. A. (2007). Tele-avaliação: validação da bateria de avaliação de leitura e escrita (BALE) on-line. (Dissertação de Mestrado). Universidade Presbiteriana Mackenzie, São Paulo, SP, Brasil.

Etchepareborda, M. C. (2003). La intervención em los transtornos disléxicos: entreinamiento de la consciência fonológica. Revista de Neurología, 36 (supp.1), 13-19.

Fletcher, J. M. (2009). Dyslexia: The evolution of a scientific concept. Journal of the International Neuropsychological Society, 15(2), 501-508.

Frith, U. (1990). Dyslexia as a developmental disorder of language. London, UK: MRC, Cognitive Development Unit.

Galaburda, A. M. \& Cestnick, L. (2003). Dislexia de dessarollo. Revista de Neurología, 36 (supp.1), 3-9.

Guimarães, S. R. K. (2003). Dificuldades no desenvolvimento da lectoescrita: o papel das habilidades metalingǘsticas. Psicologia: Teoria $e$ Pesquisa, 19(1), 33-45.

Gustafson, S. Ferreira, J. \& Ronnberg, J. (2007). Phonological or orthographic training for children with phonological or orthographic decoding deficits. Dyslexia, 13(3), 211-229.

Lukasova, K., Oliveira, D. G., Barbosa, A. C. C. \& Macedo, E. C. (2008). Habilidades de leitura e escrita de crianças disléxicas e boas leitoras. Arquivos Brasileiros de Psicologia, 60(1) 45-54.

Macedo, E. C., Capovilla, F. C., Nikaedo, C. C., Orsati, F. T., Lukasova, K., Capovilla, A. G. S. \& Diana, C. A. (2005). Teleavaliação da habilidade de leitura no ensino infantil e fundamental. Psicologia Escolar e Educacional, 9(1) 127-134.

Nikaedo, C. C. (2007). Intervenção coletiva com programa alfabetização fônica computadorizada em escolares do ensino fundamental. (Dissertação de Mestrado). Universidade Presbiteriana Mackenzie, São Paulo, SP, Brasil.

Odegard, T. N., Ring, J., Smith, S., Biggan, J. \& Black, J. (2008). Differentiating the neural response to intervention in children with developmental dyslexia. Annals of Dyslexia, 58(1), 1-14.

Oliveira, D. G. \& Macedo, E. C. (2007, setembro). Estudo do efeito de treino de consciência fonológica nas habilidades de leitura e escrita em crianças com dislexia do desenvolvimento. Anais da III Jornada de Iniciação Científica PIBIC e PIVIC da Universidade Presbiteriana Mackenzie, São Paulo, SP, Brasil.

Rashotte, C. A., MacPhee, K. \& Torgesen, J. K. (2001). The effectiveness of a group reading instruction program with poor readers in multiple grades. Learning Disability Quarterly, 24(2), 119-134.

Salgado, C. A. \& Capellini, S. A. (2008). Programa de remediação fonológica em escolares com dislexia do desenvolvimento. Pro Fono Revista de Atualização Cientifica, 20(1), 31-36.

Share, D. (1995). Phonological recoding and selfteaching: sine qua non of reading acquisition. Cognition, 55(2), 151-218.

Shastry, B. S. (2007). Developmental dyslexia: an update. Journal of Human Genetics, 52(1), 104-109.

Shaywitz, S. E., Morris, R. \& Shaywitz, B. A. (2008). The education of dyslexic children from childhood to young adulthood. Annual Review of Psychology, 59(1), 451-475.

Smythe, I., Everatt, J. \& Salter, R. (2004). International book of dyslexia: a guide to practice and resources. Chichester: John Wiley.

Torgesen, J. K. (2001). The theory and practice of intervention: comparing outcomes from prevention and remediation studies. Em: A. J. Fawcett. (Org.). Dyslexia Theory and Good Practice. (pp. 185-202). London: Whurr Publishers.

Wanzek, J. \& Vaughn, S. (2008). Response to varying amounts of time in reading intervention for students with low response to intervention. Journal of Learning Disabilities, 41(2), 126-142.

Recebido em março de 2010 Reformulado em maio de 2010 Aprovado em setembro de 2010 
Sobre os autores:

Darlene Godoy de Oliveira é psicóloga, mestranda em Distúrbios do Desenvolvimento pela Universidade Presbiteriana Mackenzie.

Katerina Lukasova é psicóloga, mestre em Distúrbios do Desenvolvimento pela Universidade Presbiteriana Mackenzie e professora do curso de Psicologia na Universidade Cruzeiro do Sul.

Elizeu Coutinho de Macedo é psicólogo, mestre e doutor em Psicologia Experimental pela Universidade de São Paulo e professor do Programa de Pós-Graduação em Distúrbios do Desenvolvimento da Universidade Presbiteriana Mackenzie.

Estudo realizado em Projeto de Iniciação Científica de Darlene Godoy de Oliveira, com financiamento do MackPesquisa. 